\title{
Advanced Methodologies for Atomic-Scale Nanofabrication and Dynamic Characterization
}

\author{
Xing Wu and Litao Sun ${ }^{\S}$ \\ SEU-FEI Nano-Pico Center, Key Laboratory of MEMS of Ministry of Education, \\ School of Electronic Science and Engineering, Southeast University, Nanjing, 210096, P. R. China \\ ${ }^{\S}$ Email: slt@,seu.edu.cn
}

\begin{abstract}
Based on the idea of "setting up a nanolab inside a transmission electron microscopy (TEM)", we review our recent progress in atomic resolution nanofabrication and dynamic characterization of individual nanostructures and nanodevices. The electron beam can be used as a tool to induce nanofabrication on the atomic scale. Additional probes from a special-designed holder provide the possibility to further manipulate and measure the electrical properties of the nanostructures. All phenomena from the in-situ TEM experiments can be recorded in real time with atomic resolution.
\end{abstract}

Keywords - Transmission electron microscopy, Dynamic characterization, Nanofabrication, Nanodevice.

\section{INTRODUCTION}

Dynamic characterization technique at atomic-scale is important over a wide range of areas in science and technology, including low-dimensinoal nanomaterials in modern nanotechnology, nanoscale fabrication and electrical measurement for nanodivce. Electron microscopy opens a unique window into structures and processes, as it provides a combination of temporal and spatial resolution that is not achievable with other techniques. To apply an external field such as strain, electrical, or thermal fields, special experimental setups in the specimen chamber of a transmission electron microscope should be designed. However, the space between the pole pieces in the object lens of a commercial transmission electron microscope is usually less than $5 \mathrm{~mm}$, which limits the dimensions of in-situ test instruments. To solve this problem, special types of dedicated functional transmission electron microscopy (TEM) specimen holders (hereafter denoted as TEM holders) with built-in integrated micro-electromechanical systems (MEMS) have been developed. These TEM holders are now commercially available for several applications such as heating, electrical probing, straining, or mechanical testing. In contrast with in-situ scanning electron microscopy (SEM), in-situ TEM has a higher spatial resolution and can enable the acquisition of complete information on a specimen, not only surface information. Continuous efforts in electron optics, particularly the invention of aberrationcorrection devices, are leading to the reduction of the lateral resolution of transmission electron microscopes to subangstrom regime, which is already smaller than the distance between atoms in densely packed crystals. With the development of strongly focused electron probes, TEM can also be used with energy-dispersive spectroscopy (EDS) and electron energy loss spectroscopy (EELS) to enable highspatial-resolution chemical analyses and elemental mapping of a specimen before/during and after testing.

The layout of this paper is as follows. In Section II, we present the indirect loading on nanomaterial with electron beam irradiation induced high pressure. This will be followed by our nanostructure applications in Section III that point to the subnanometer fabrication of nanowire and nanopore. Recent observations of conductive filament formation and rupture in novel memory device will be presented in Section IV. Finally, we conclude with a summary of the presented results and propose ideas for further exploratory work on modern in-situ TEM.

\section{INDIRECT LOADING ON NANOMATERIALS: IRRADIATION INDUCED HIGH PRESSURES}

In general, electron irradiation is inevitable in electron microscopy because the image of a specimen is the result of the interaction between an electron beam and the specimen atoms. In a typical TEM system, the acceleration voltage ranges from $80 \mathrm{kV}$ to $300 \mathrm{kV}$, which is sufficiently high to displace atoms in the specimen and leads to visible structural transformation. The electron beam scattering in the specimen can be classified as elastic or inelastic. In insulators, the inelastic scattering between electron beam and electron clouds in a specimen can induce electronic excitations, resulting in bond breakage and lattice reconstruction. However, in solids, conduction electrons quickly quench electronic excitations. Thus, the main damage in conductive solids is due to ballistic atom displacement, which can be regarded as the collision between an electron and a nucleus (1). This process resembles the Compton scattering in physics.

The energy $T$ that is transferred to the nucleus depends on the scattering angle $\theta$ :

$$
T(\theta)=T_{\max } \cos ^{2} \theta,
$$

where $T_{\max }$ is the maximum energy when a head-on collision $\operatorname{occurs}(\theta=0)$. 
If the law of momentum conservation is applied, we can derive the expression for $T_{\max }$ as a function of the electron energy $E$ :

$$
T_{\max }=2 E\left(E+2 m_{\mathrm{e}} c^{2}\right) / M c^{2}
$$

where $m_{\mathrm{e}}, M$, and $c$ denotes the electron mass, mass of nucleus, and speed of light, respectively. Indeed, head-on collisions are rare and large-angle scattering frequently occurs.

Then, we introduce the displacement threshold energy, which determines the occurrence of a displacement accident. Above this threshold energy, the displaced atoms are knocked onto an interstitial site and leave a vacancy in the lattice. These vacancy-interstitial pair cannot be annihilated and a persistent defect is generated. For single-walled carbon nanotubes (CNTs), the threshold electron energy is $86 \mathrm{keV}$, which corresponds to an energy of $16 \mathrm{eV}$ that has to be transferred to a carbon atom for displacement. On the other hand, for multiwalled CNTs, the threshold electron energy is $100 \mathrm{keV}$. Fullerene molecules are more sensitive and tend to coalesce, forming a large cage at a threshold energy of $<80 \mathrm{keV}$ (1).

Interestingly, graphitic materials have a unique ability to reconstruct after vacancy generation. In a graphene sheet, monovacancies have a low migration energy $(1.0-1.6 \mathrm{eV})$, whereas divacancies are almost immobile $(7 \mathrm{eV})$. Therefore, monovacancies rapidly migrate and tend to form immobile and stable divacancies. Defect agglomeration is important because monovacancies cannot be removed by lattice reconstruction, whereas divacancies can vanish by the closure of highly energetic dangling bonds. Irradiation-induced sustained mass loss results in decreased surface area of graphene accompanied by a local change in curvature because only the hexagonal lattice is flat, whereas pentagons introduce positive curvature and heptagons introduce negative curvature. The shrinkage of carbon shells is essential and can be used to achieve high pressure in TEM, as described in the following section. Notably, the aforementioned reconstruction mechanism can only occur when each monovancancy is sufficiently mobile to coalesce with another vacancy. Therefore, the temperature of a specimen should be increased. Under a typical irradiation condition, the critical temperature for reconstruction should range from $200{ }^{\circ} \mathrm{C}$ to $300{ }^{\circ} \mathrm{C}$, which can easily be reached in the heating stage inside the TEM.

In 2008, using the above high pressure mechanism, an artful experiment to observe the plastic deformation of a nanometer-sized metallic crystal lattice inside carbon onions encapsulating metal crystal was designed, as shown in Figure $1(2,3)$. In this experiment, the spherical graphitic shells are initially punctured by focusing an electron beam, thereby leaving a nanohole. Gold crystals are then extruded out of graphitic cages at a high pressure. Through a direct comparison of the lattice spacings in gold nanocrystals inside and outside the cages, a high pressure of $20 \mathrm{GPa}$ is found at $300{ }^{\circ} \mathrm{C}$. Considering that the ideal shear strength of gold is only $1 \mathrm{GPa}$, the high pressure inside this cage is extremely high. However, dislocation is not observed, although occasional grain boundaries and planar stacking faults appear. One possible explanation is that the transient dislocations nucleate and vanish so rapidly that they cannot be captured by TEM. Although this experiment is carried out at $300{ }^{\circ} \mathrm{C}$, molecular simulations demonstrate that the observed plastic deformation is a result of nucleation and motion of short-lived dislocations, not of the diffusion of atoms.
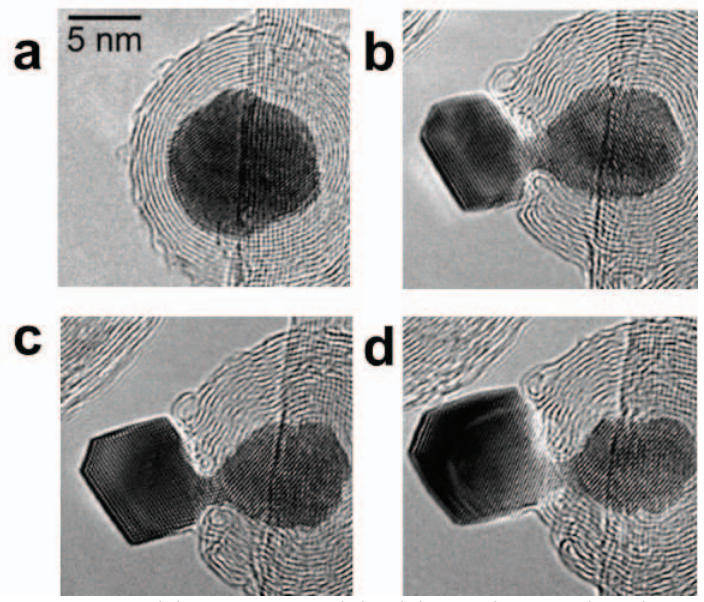

Figure 1 A gold nanocrystal inside carbon onion is extruded from the punctured location under irradiation. Irradiation times: (a) $0 \mathrm{~s}$, (b) $540 \mathrm{~s}$, (c) $660 \mathrm{~s}$, and (d) $900 \mathrm{~s}$. (Temperature: $\left.300^{\circ} \mathrm{C}\right) \cdot(2)$

When a high pressure builds in CNTs filled with metallic or carbide nanowires, some phenomena different from carbon onions occur. These phenomena can be attributed to the free space inside the tubes. Figure 2 shows that a cobalt nanowire inside a collapsing CNT under irradiation is severely deformed and acquires a bottleneck shape (4). As pointed out above, tube shrinkage occurs when high pressure is exerted in the radial direction onto the encapsulated metal nanowires. By measuring the decrease in lattice spacing in cobalt, a high pressure of about $40 \mathrm{GPa}$ is observed. Under this high pressure, the metal or carbide nanowires can be thinned and extruded in the axial direction of the tube because of Poisson's effect. Thus, this experiment demonstrates for the first time that CNTs can be utilized as gigapascal high-pressure cells to deform hard materials, i.e., the nanoextruder effect (4-6). A similar high-pressure experiment has been carried out in a diamond anvil cell, in which two perfectly aligned gem diamond anvil squeezes a sample loaded in the gasket hole. The diamond anvil cell method must be combined with X-ray diffraction and Raman spectroscopic measurements to estimate structural variation. However, this process is not in real time and thus cannot enable the determination of the detailed structure deformation mechanism. CNTs are electron transparent, so all detailed structural changes in fillings such as defect formation can be visible. An electron beam not only induces the high pressure to deform the metal crystal but also provides images with lattice resolution during such deformation. Furthermore, the deformation rate of fillings (in 
the range of $1 \mathrm{~nm} / \mathrm{s}$ ) can be controlled by tuning the electron beam intensity. A later work (7) shows that such a high pressure can be released by vacancies in CNTs caused by subsequent irradiation under room temperature. Thus, a full pressure loop (i.e., loading and unloading) on a nano-object is achieved in TEM. This finding opens an opportunity for the direct observation of the atomic-scale development of highpressure-induced phenomenon, or the so-called in-situ highpressure electron microscopy.
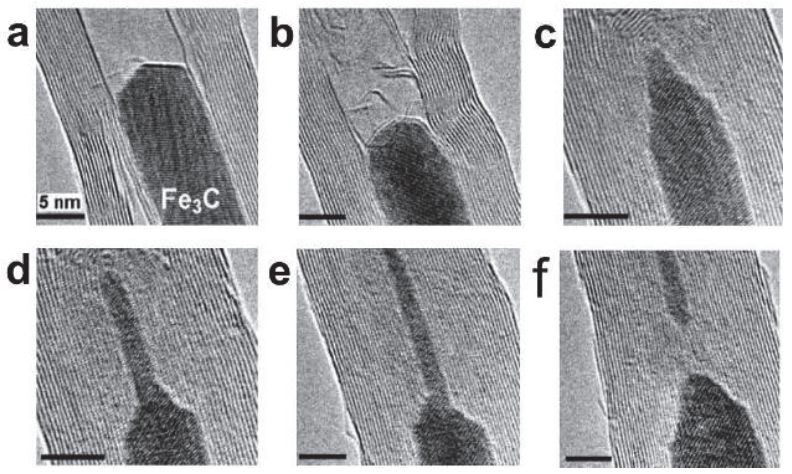

Figure 2 Evolution of a multi-walled CNTs partly filled with a $\mathrm{Fe}_{3} \mathrm{C}$ nanowire under electron irradiation with an electron beam during a total period of $50 \mathrm{~min}$ at a specimen temperature of $600{ }^{\circ} \mathrm{C}$. (a) Tube before irradiation. (b) to (e) Irradiation leads to a collapse of the tube and deformation of the $\mathrm{Fe}_{3} \mathrm{C}$ crystal. (f) Tube collapse cuts off the thinned $\mathrm{Fe}_{3} \mathrm{C}$ crystal. (4)

\section{IRRADIATION DERIVED NANOSTRUCTURE FABRICATION}

In-situ electron irradiation in a transmission electron microscope is also a helpful approach to fabricate uniform, robust semiconducting molybdenum-sulfide $\left(\mathrm{MoS}_{2}\right)$ ribbon with sub-nanometer width. It is shown that the sulphur atoms at the edge of a $\mathrm{MoS}_{2}$ sheet are too fragile to withstand the electron beam irradiation, so that holes of defects, once being created, can rapidly spread towards each other and produce ribbons between them. When the ribbon width is narrowed to a critical size well below one nanometer, phase transition into a distinctly robust $\mathrm{Mo}_{5} \mathrm{~S}_{4}$ structure occurs spontaneously before the holes coming into coalescence.

Our in-situ electron irradiation experiment starts with a monolayer $\mathrm{MoS}_{2}$ sample prepared by micromechanical cleavage as identified by both optical (Figure 3a) and high resolution transmission electron microscope (HRTEM) characterization (Figures. $3 b$ and $3 c$ ). Under the irradiation of electron beam, defects can be created in the atomic thin 2dimensional sheet once the beam energy exceeds the knock-on damage threshold of the sheet (8). As the maximum energy transferred from an $80 \mathrm{kV}$ electron to a sulphur atom is about $6 \mathrm{eV}$, between the thresholds of the edge and interior sulphur vacancies of the $\mathrm{MoS}_{2}$ sheet, we chose an acceleration voltage of $80 \mathrm{kV}$ to achieve enough resolution while maintain the
$\mathrm{MoS}_{2}$ sheet in highly crystalline. The electron beam was firstly focused to $40 \mathrm{~A} / \mathrm{cm}^{2}$ to induce initial defects and holes in the $\mathrm{MoS}_{2}$ sheet. After 81 seconds irradiation, small vacancy aggregations formed as labelled by the arrows in Figure 3d. As the edge of the defects is much weaker, all the defects spread rapidly in exposure to the irradiation. After another $10^{3}$ seconds irradiation, the defects extended into holes with diameters of $3 \sim 6 \mathrm{~nm}$ as shown in Figure 3e, with an average edge shrinking speed of about $0.02 \mathrm{~nm} / \mathrm{s}$. Along with the growth of holes, the connecting regions between them were narrowed into nanoribbons, showing a trend for coalescence. But just before the coalescence occurred, the thinnest connecting ribbon spontaneously turned into a fine ribbon with smooth edges and uniform width of $0.35 \mathrm{~nm}$ (Figure $3 \mathrm{f}$ ), which was robust to the dense electron irradiation. To carefully investigate the surprising fine ribbon, we decreased the beam intensity to $10 \mathrm{~A} / \mathrm{cm}^{2}$. The typical formation and elongation process for a fine ribbon was recorded in Figures $3 \mathrm{~g}$ to 31 . The formed ribbon showed uniform width with identical atomic structures, in sharp contrast to the mother $\mathrm{MoS}_{2}$ at its ends.

In-situ HRTEM characterization combining with firstprinciples calculations identifies the sub-nanometer ribbon as a $\mathrm{Mo}_{5} \mathrm{~S}_{4}$ crystal derived from $\mathrm{MoS}_{2}$ through a spontaneous phase transition (9). Further first-principles investigations show that the $\mathrm{Mo}_{5} \mathrm{~S}_{4}$ ribbon has a bandgap of $0.77 \mathrm{eV}$ and can bear $9 \%$ tensile strain before breaking with a Young's modulus of $300 \mathrm{GPa}$. Figure 4 shows the roadmap of semiconductor industry (10) and nanotechnology community to manufacture narrower functional nanostructures. The development in micro-nanofabrication technologies are propelling the dimension of flash down to 10-nanometers, but facing great challenges in manufacture at sub-10-nanometer scale. On the other hand, CVD grown (11), STM tailored (12) and sonochemical derived $(13,14)$ graphene nanoribbons (GNRs) can be ranged well into sub-10-nanometers, but the distributions of widths are quite scattered as shown by the arrows in Figure 4 and the edges are rough when the ribbon width is down to $5 \mathrm{~nm}$ (15). Distinctively, the irradiationderived robust sub-nanometer $\mathrm{Mo}_{5} \mathrm{~S}_{4}$ ribbon has a unique width and bandgap with smooth edges, which are vital to serve as built blocks for functional devices. Moreover, if the initial defects and holes in a $\mathrm{MoS}_{2}$ layer can be induced in a controlled manner, it will be also possible to fabricate ordered array or networks of the sub-nanometer ribbons. Therefore, there are still a lot of attractive possibilities waiting for attempts along this route for a blueprint toward producing subnanometer electronics.

Electron irradiation can also be used to fabricat solidstate (embedded in silicon nitride (16), silicon oxide (17), or aluminum oxide (18) membranes) nanopore to detect singlestrand DNA or RNA. Figure 5 shows that the nanopores were fabricated on the graphene membranes by a highly focused electron beam. During nanopore fabrication, the electron beam was carefully adjusted and quickly moved to a fresh region on 
the graphene sheet at a pre-defined irradiation time less than $20 \mathrm{~s}$. When the fabrication process was complete, the electron beam was quickly sheltered and then widely distributed to minimize irradiation damage during routine imaging. The nanopore temperature was maintained at above $400{ }^{\circ} \mathrm{C}$ for at least $30 \mathrm{~min}$ without irradiation. Finally, the nanopores with sizes comparable to those of the as-fabricated nanopores were imaged by a spread electron beam to determine whether shrinkage or expansion has occurred. Figure 5b-c show the typical shrinkage of graphene nanopores with an initial

diameter of $\sim 3.8 \mathrm{~nm}$ at $400{ }^{\circ} \mathrm{C}$, while Figure $5 \mathrm{~d}$-e show the

expansion of nanopores with an initial diameter of $\sim 7.8 \mathrm{~nm}$ at

$400{ }^{\circ} \mathrm{C}$. This approach could be developed into a general controllable method for fabrication of sub-nanometer building blocks from low-dimensional nanostructures for future functional devices.
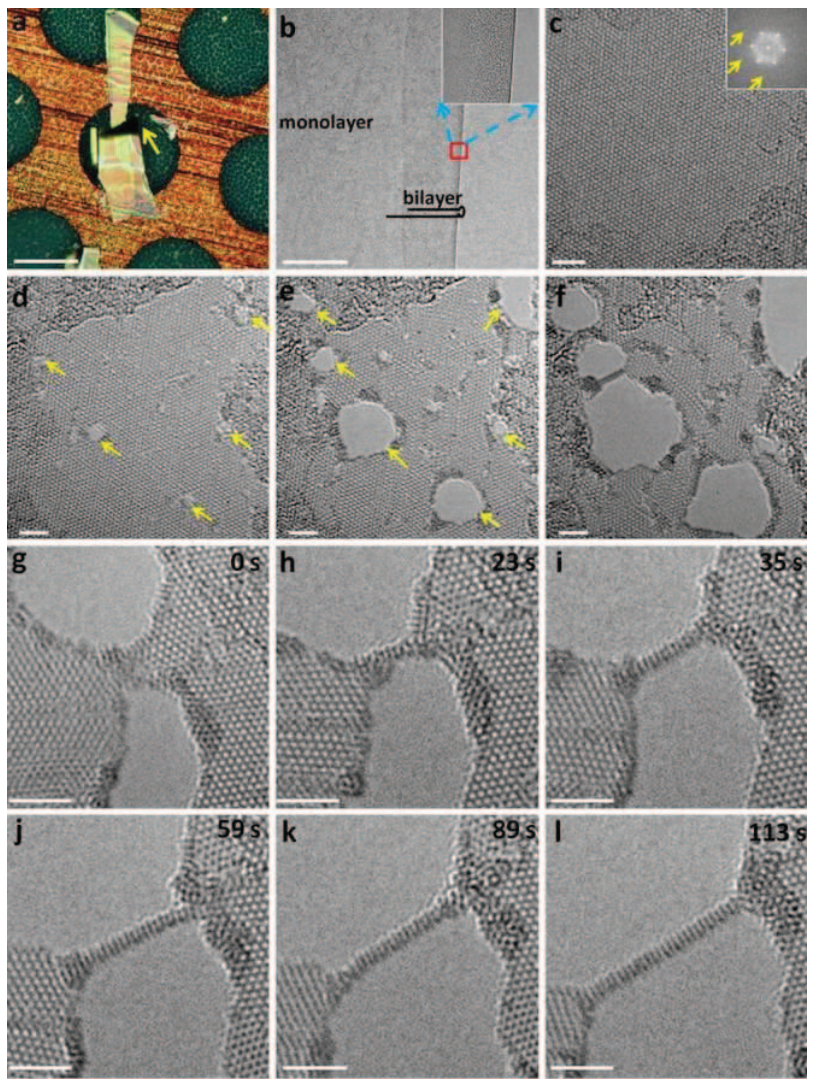

Figure 3 In-situ fabrication of suspended molybdenum sulfide sub-nanometer ribbons from monolayer $\mathrm{MoS}_{2}$ by electron irradiation. (a) A few layer $\mathrm{MoS}_{2}$ flake on TEM grid. The thinnest region locates at the region labeled by arrow. (b) TEM images of the thinnest region. The thickness of the membrane can be determined by fringe counting at the edge. The straight edge verifies that the membrane is single layer. The folded and unfolded region can be further determined by contrast. (c) HRTEM data obtained on the unfolded regions. The corresponding FFT image is shown in the inset. (d) The initial $\mathrm{MoS}_{2}$ membrane with small irradiation-induced vacancies as highlighted by the arrows. (e-f) Larger holes extended from the small vacancies upon $80 \mathrm{kV}$ electron irradiation of another 108 and 261 seconds. (g-l) Time series of the formation and growth for a suspended 1D structure under $80 \mathrm{kV}$ electron irradiation. The scale bar is $1 \mathrm{~nm}$ in (gl). (9)

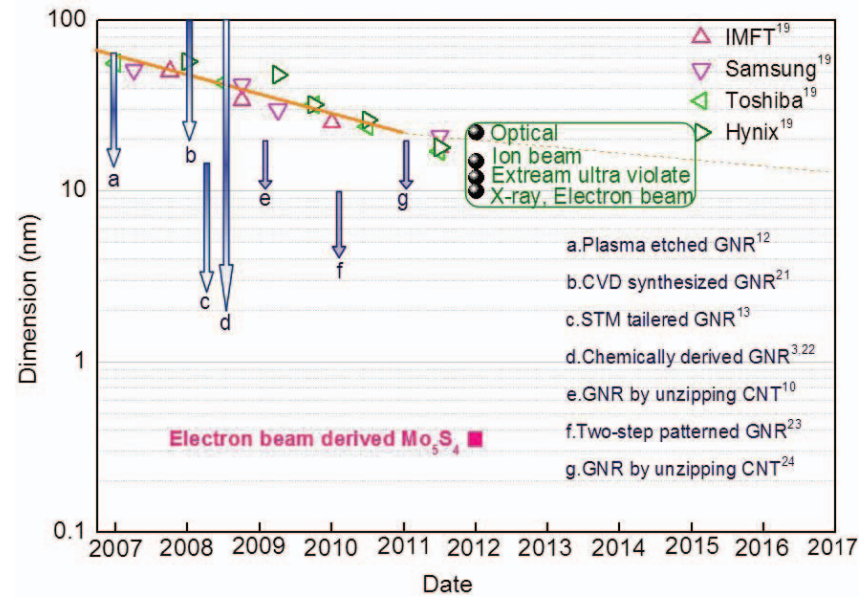

Figure 4 Dimension distributions of flash $1 / 2$ pitch, lithography resolutions, width of GNRs and $\mathrm{Mo}_{5} \mathrm{~S}_{4}$ ribbon. The triangles and solid circles stand for the reported dimension of flash $1 / 2$ pitch (11) and lithography resolutions. The solid and dot lines are the time lines of flash industry (10).

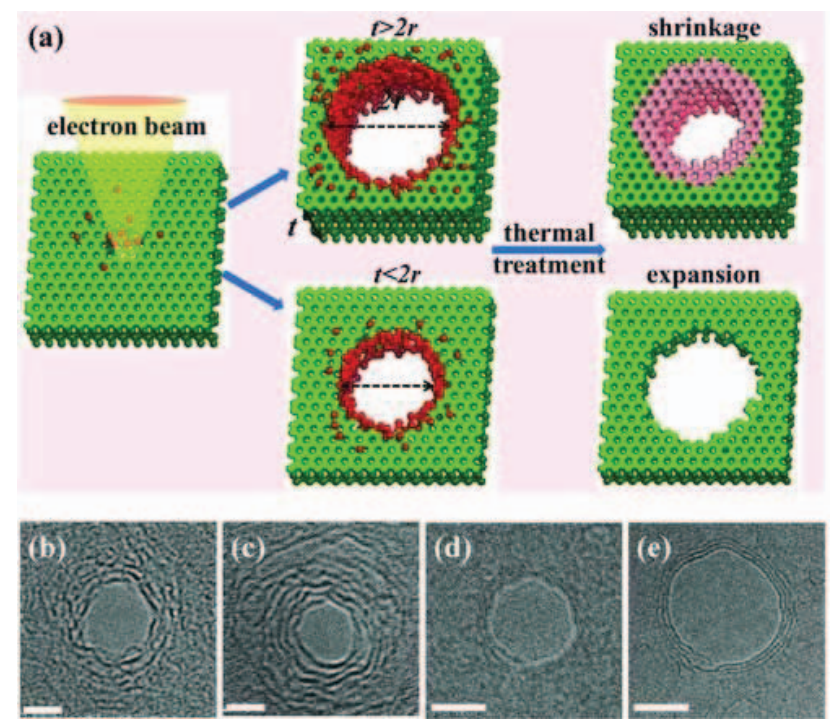

Figure 5 TEM image of the fabrication and evolution 
processes of graphene nanopores: (a) Schematic of the experiment illustrating the fabrication and evolution processes of graphene nanopores and (b-e) TEM images showing nanopore shrinkage $(\mathbf{b}-\mathbf{c})$ or expansion $(\mathbf{d}-\mathbf{e})$ under thermal excitation. The scale bar is $2 \mathrm{~nm}$ in (b-c) and $5 \mathrm{~nm}$ in (d-e). (19)

\section{DIRECT LOADING ON NANODEVICE: ELECTRICAL MEASUREMENT}

Electrical measurement of nanodevice can also be performed inside TEM. The key benefits of in-situ TEM electrical probing techniques lie in the abilities to: (1) locate and align a probe to nanoscale objects with high precision; (2) characterize both object and probe by high resolution imaging using TEM; (3) carry out electrical and mechanical probing in situ; (4) study dynamical processes.

$\mathrm{ZrO}_{2}$-based resistive random-access memory (RRAM) devices with various metal electrodes: $\mathrm{Cu}, \mathrm{Ag}$, and $\mathrm{Ni}$ (20-22). Devices are prepared on the platform of a $\mathrm{W}$ probe and a $\mathrm{SiO}_{2} / \mathrm{Si}$ substrate by successively depositing a $10 \mathrm{~nm}$ thick Ti adhesion layer, a $50 \mathrm{~nm}$-thick Pt bottom electrode layer, a 40 $\mathrm{nm}$ thick $\mathrm{ZrO}_{2}$ film and a $70 \mathrm{~nm}$ metal top electrode layer using electron beam evaporation. The $\mathrm{Cu}$ (or $\mathrm{Ag}, \mathrm{Ni}) / \mathrm{ZrO}_{2} / \mathrm{Pt}$ devices on the $\mathrm{SiO}_{2} / \mathrm{Si}$ substrate with $100 \times 100 \mu \mathrm{m}^{2}$ cell area are fabricated by the lithography and lift-off processes. The $\mathrm{Cu}$ (or $\mathrm{Ag}, \mathrm{Ni}$ ) $/ \mathrm{ZrO}_{2} / \mathrm{Pt}$ stacked films on the platform of the $\mathrm{W}$ probe is fabricated to multiple TEM specimens with small lateral sizes $(\sim 200 \mathrm{~nm})$ by using a dual-beam focused ion beam (FIB) system, as shown in Figure 6.

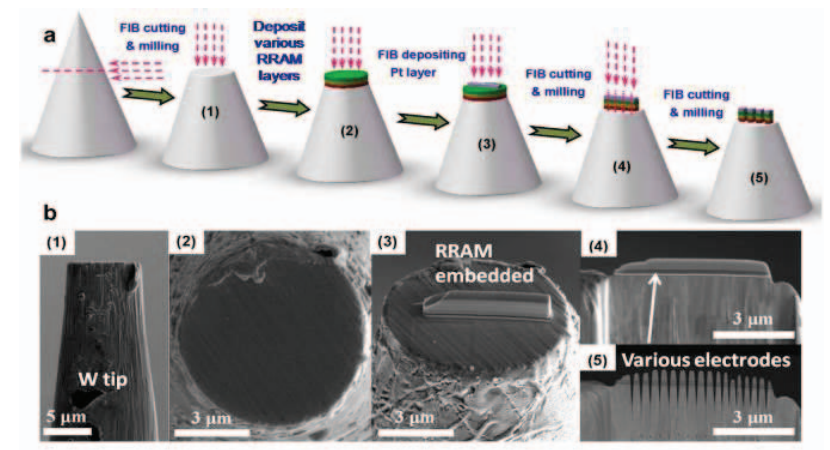

Figure 6 In situ fabrication technique used to produce RRAM TEM specimen. (a) Schematic of the methods used to fabricate the Ag (or $\mathrm{Cu}, \mathrm{Ni}) / \mathrm{ZrO}_{2} / \mathrm{Pt}$ TEM specimen. (b) Corresponding SEM images in each of the TEM specimen fabrication processes. Images (1)-(2) show a cross-sectional of $\mathrm{W}$ platform and a top-view of the platform with RRAM multi-layer, indicating the platform has a smooth surface to meet the requirement for fabricating the RRAM device. Images (3)-(5) confirm that multiple TEM specimens with small size RRAM structure can be achieved through this method. (21)

Figures 7a-7e show the growth dynamics of conductive filaments (CFs) between the $\mathrm{Cu}$ and $\mathrm{Pt}$ electrodes under a $-4 \mathrm{~V}$ constant voltage stress, with a $1.1 \mu \mathrm{A}$ compliance current $\left(I_{C C}\right)$ applied to the Pt electrode. These images were extracted from a $140 \mathrm{~s}$ video, with images a-e representing frame times at 0 , $60,110,120$ and $130 \mathrm{~s}$, respectively. In the video images, a first CF suddenly emerged across the $\mathrm{ZrO}_{2}$ film between the two electrodes at $\sim 60 \mathrm{~s}$. The sudden appearance of the first CF was accompanied by an equally sudden increase in the electrical current through the device (top figure). However, the low resistance state was only maintained for a short time, probably because of the instability of this particular $\mathrm{CF}$, which led to a spontaneous rupture. When the stress time reached $110 \mathrm{~s}$, the second CF began to form inside the $\mathrm{ZrO}_{2}$ film and appeared to be connected only to the $\mathrm{Cu}$ electrode. From $110 \mathrm{~s}$ to $130 \mathrm{~s}$, the second $\mathrm{CF}$ continued to grow until reaching the Pt electrode (Figure 7c-7e). During this process, the electrical current continued to increase and finally jumped to the value of $I_{C C}$ (top figure).

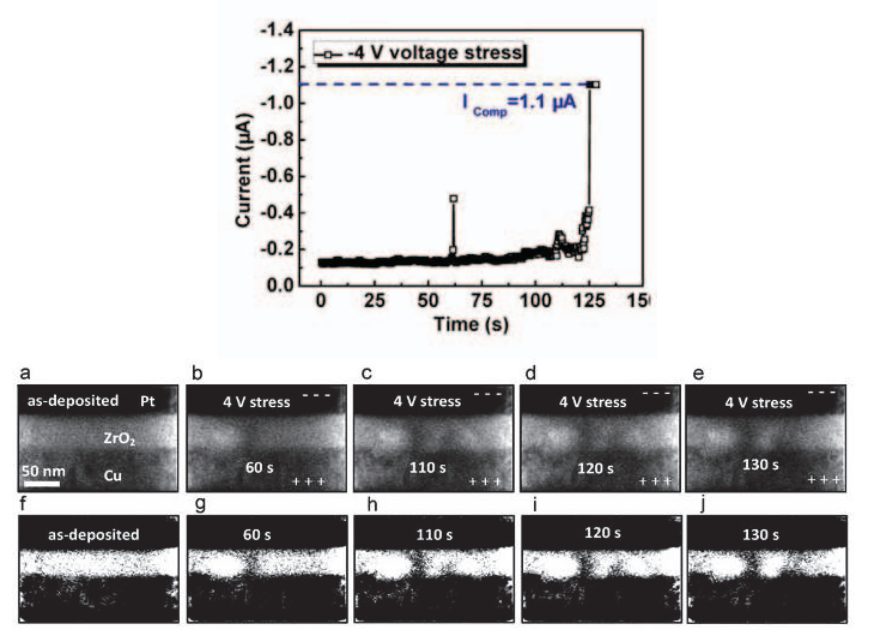

Figure 7. Top: Current versus time curve of the TEM specimen under a $-4 \mathrm{~V}$ constant stress. (a)-(e) Dynamics of CF growth. A series of TEM images capturing the dynamic $\mathrm{CF}$ growth processes in the $\mathrm{Cu} / \mathrm{ZrO}_{2} / \mathrm{Pt}$ TEM device. (f)-(j) Black-and-white images converted from the raw TEM images of the (a)-(e) to highlight the filaments. (21)

Beside metal oxide-based RRAM, antoher type of RRAM device is based on Ag-Ge-Se (AGS) electrolytes. The dynamic evolution of conductive phases in AGS is also studied via in- situ TEM technique. The glassy AGS samples used for TEM observations were deposited via pulsed laser deposition technique. AGS films of $100 \mathrm{~nm}$ thick were grown on well-polished $\mathrm{NaCl}$ single crystal substrates at room temperature. Then we put these as-grown samples into deionized water to dissolve $\mathrm{NaCl}$ substrates and carefully dredged up the AGS films using copper grids. This keeps the AGS samples away from those unwanted damages by ion milling or FIB cutting because AGS are unstable against these treatments. The copper girds were cut in half, insuring some part of AGS film being exposed outside for the following electrical contact. The rest of the film is still on the copper girds, where the grids were grounded as an inert electrode. The prepared samples were then equipped on a in-situ TEM holder that supplies an Ag tip as active electrode. Figure 8a shows the 
schematic of the in-situ experimental set-up. Biases are applied on this Ag tip for the in-situ observations.

The movable holder is driven carefully toward the Ag tip till the tip contacts the AGS film. This whole contacting process is monitored via TEM. The final contact structure of the grid/AGS/Ag tip is shown in Figure 8b. Most part of the sample is flat and homogeneous except for some wrinkles near the grid. The as-prepared AGS is initially in its amorphous state, as confirmed by the selected area electron diffraction (SAED) pattern (the inset of Figure 8b). Then a positive bias of $0.5 \mathrm{~V}$ is applied onto the $\mathrm{Ag}$ tip to trigger the oxidation of Ag. Here a small bias and a wide span of $600 \mathrm{~nm}$ between the tip and grid are just for the slow down of the memristive transition, which facilitates us to capture the detailed structural evolution.

On loading the bias, the active Ag electrode is electrochemically oxidized. The growth of conductive pathways takes place as $\mathrm{Ag}^{+}$ions keep injecting into the electrolyte. The whole process was recorded and summarized in Figures $8 \mathrm{c}$ to $1 \mathrm{j}$. However, this AGS device does not work the way that other electrolytic devices (like $\mathrm{Ag}_{2} \mathrm{~S}$ ) do. The conductive dendrites grow in opposite directions. The growth of the filament has been reported to be limited by the cation transport process (23). In those electrolytic devices with high cation mobility, Ag dendrites should grow from the cathode toward the $\mathrm{Ag}$ electrode because $\mathrm{Ag}^{+}$ions reach the cathode and become reduced there in the light of the conventional electrochemical metallization theory $(24,25)$. But Figures $8 \mathrm{c}$ and $8 \mathrm{~d}$ indicate that the nucleation of dendrites in AGS electrolyte starts from Ag electrode, as marked by the yellow circles, which is unexpected in previous studies. Further, it is $\mathrm{Ag}_{2} \mathrm{Se}$ precipitate not $\mathrm{Ag}$ reduction product that composes the conductive dendrite. The SAED pattern of the dendrite is shown in Figure 8k, which identifies the dendrite is composed of orthorhombic $\mathrm{Ag}_{2} \mathrm{Se}$ with symmetry of P212121. Corresponding $(0 \overline{3} 1),(0 \overline{1} 3)$ and $(0 \overline{2} \overline{2})$ lattice constants are calculated to be $0.225,0.242$ and $0.261 \mathrm{~nm}$, respectively.

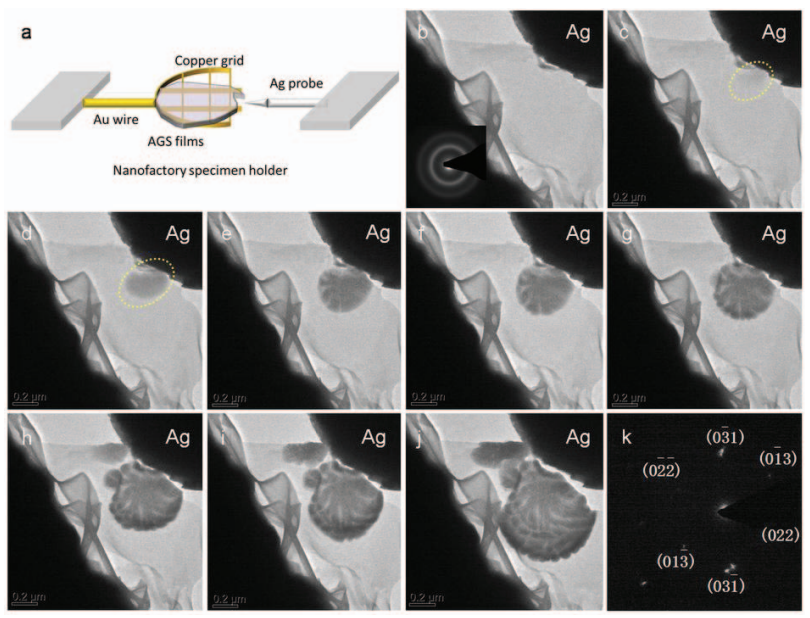

Figure 8 Structural evolution caused by the chemically driven phase transformation. (a) Schematic of the in-situ experimental set-up. (b-j) TEM images showing the growth of dendrites. (k) SAED pattern demonstrating the dendrites composed of orthorhombic $\mathrm{Ag}_{2} \mathrm{Se}$. (26)

\section{CONLUSIONS AND OUTLOOK}

The in-situ TEM studies of low-dimensional nanomaterial and novel nanodevice promote our understanding of physical mechanism. These works are necessary for the applications of low-dimensional nanomaterials and novel nanodevice in modern nanotechnology. Several possible future directions of in-situ TEM are noted as follows:

(1) The spatial resolution of modern TEM has already been extended to below the $50 \mathrm{pm}$ with the development of the aberration correction technique. Although the spatial resolution can still be enhanced, the limiting resolution in TEM is approaching. On the other hand, the factor of time may be a breakthrough point for the advancement of in situ TEM. Using pulsed electron beams from a photocathode illuminated by a pulsed laser $(27,28)$, the dynamic process on a time scale of $10^{-13} \mathrm{~s}$ has been captured. Ultrafast TEM now requires a special external setup and is not commercialized. Considering that many physical processes occur in the range of $10^{-13} \mathrm{~s}$, the revolution of ultrafast TEM applications is conceivable.

(2) Multiple prodes should be integrated into TEM to acquire more data. For example, the standard four-terminalmeasurement of sheet resistance of nanodevice can be performed if multiple probes are used.

(3) All in-situ TEM experiments on low-dimensional nanomaterials and novel nanodevice are currently conducted in a vacuum at room temperature. However, their mechanical properties vary at different temperatures and environments. The mechanical properties of these materials under cooling, heating treatments, or under a gas environment need further study.

\section{ACKNOWLEDGMENT}

This work was supported by the 973 programs (2011CB707601), Jiangsu Province Funds for Distinguished Young Scientists (No. BK2012024), and Chinese postdoctoral funding (No. 2012M520053).

\section{REFERENCES}

1. F. Banhart, "Irradiation effects in carbon nanostructures." Reports on Progress in Physics 62, 1181 (1999).

2. L. Sun, A. V. Krasheninnikov, T. Ahlgren, K. Nordlund, F. Banhart, "Plastic deformation of single nanometer-sized crystals." Physical review letters 101, 156101 (2008).

3. S. Suresh, J. Li, "Deformation of the ultra-strong." Nature 456, 716 (2008). 
4. L. Sun, F. Banhart, A. Krasheninnikov, J. RodriguezManzo, M. Terrones, P. Ajayan, "Carbon nanotubes as high-pressure cylinders and nanoextruders." Science 312, 1199 (2006).

5. J. A. Rodríguez-Manzo, M. Terrones, H. Terrones, H. W. Kroto, L. Sun, F. Banhart, "In situ nucleation of carbon nanotubes by the injection of carbon atoms into metal particles." Nature nanotechnology 2, 307 (2007).

6. Z. Wang, Y. Zhao, "High-pressure microscopy." Science 312, 1149 (2006).

7. M. D. Ma, J. Z. Liu, L. Wang, L. Shen, L. Xie, F. Wei, J. Zhu, Q. Gong, J. Liang, Q. Zheng, "Reversible highpressure carbon nanotube vessel." Physical Review B 81, 235420 (2010).

8. H.-P. Komsa, J. Kotakoski, S. Kurasch, O. Lehtinen, U. Kaiser, A. V. Krasheninnikov, "Two-dimensional transition metal dichalcogenides under electron irradiation: defect production and doping." Physical review letters 109, 035503 (2012).

9. X. Liu, T. Xu, X. Wu, Z. Zhang, J. Yu, H. Qiu, J.-H. Hong, C.-H. Jin, J.-X. Li, X.-R. Wang, "Top-down fabrication of sub-nanometre semiconducting nanoribbons derived from molybdenum disulfide sheets." Nature Communications 4, 1776 (2013).

10. "http://www.itrs.net/reports.html." (2013).

11. J. Campos-Delgado, J. M. Romo-Herrera, X. Jia, D. A. Cullen, H. Muramatsu, Y. A. Kim, T. Hayashi, Z. Ren, D. J. Smith, Y. Okuno, "Bulk production of a new form of $\mathrm{sp}^{2}$ carbon: Crystalline graphene nanoribbons." Nano letters 8, 2773 (2008).

12. M. Y. Han, B. Özyilmaz, Y. Zhang, P. Kim, "Energy band-gap engineering of graphene nanoribbons." Physical review letters 98, 206805 (2007).

13. X. Li, X. Wang, L. Zhang, S. Lee, H. Dai, "Chemically derived, ultrasmooth graphene nanoribbon semiconductors." Science 319, 1229 (2008).

14. X. Wang, Y. Ouyang, X. Li, H. Wang, J. Guo, H. Dai, "Room-temperature all-semiconducting sub-10-nm graphene nanoribbon field-effect transistors." Physical review letters 100, 206803 (2008).

15. L. Tapasztó, G. Dobrik, P. Lambin, L. P. Biro, "Tailoring the atomic structure of graphene nanoribbons by scanning tunnelling microscope lithography." Nature nanotechnology 3, 397 (2008).

16. J. Li, M. Gershow, D. Stein, E. Brandin, J. Golovchenko, "DNA molecules and configurations in a solid-state nanopore microscope." Nature materials $\mathbf{2}$, 611 (2003).

17. H. Chang, F. Kosari, G. Andreadakis, M. Alam, G. Vasmatzis, R. Bashir, "DNA-mediated fluctuations in ionic current through silicon oxide nanopore channels." Nano letters 4, 1551 (2004).

18. B. M. Venkatesan, A. B. Shah, J. M. Zuo, R. Bashir, "DNA Sensing Using Nanocrystalline Surface Enhanced A12O3 Nanopore Sensors." Advanced functional materials 20, 1266 (2010).

19. T. Xu, K. Yin, X. Xie, L. He, B. Wang, L. Sun, “Size - dependent evolution of graphene nanopores under thermal excitation." Small 8, 3422 (2012).

20. Q. Liu, S. Jun, H. Lv, S. Long, L. Li, K. Yin, N. Wan, Y. Li, L. Sun, M. Liu, "Response to "comment on real - time observation on dynamic growth/dissolution of conductive filaments in oxide - electrolyte - based ReRAM” .” Advanced Materials 25, 165 (2013).

21. Q. Liu, J. Sun, H. Lv, S. Long, K. Yin, N. Wan, Y. Li, L. Sun, M. Liu, "Real-time observation on dynamic growth/dissolution of conductive filaments in oxide electrolyte - based ReRAM.” Advanced Materials 24, 1844 (2012).

22. J. Sun, Q. Liu, H. Xie, X. Wu, F. Xu, T. Xu, S. Long, H. Lv, Y. Li, L. Sun, "In-situ observation of nickel as an oxidizable electrode material for the solid-electrolytebased resistive random access memory." Applied Physics Letters 102, 053502 (2013).

23. Y. Yang, P. Gao, S. Gaba, T. Chang, X. Pan, W. Lu, "Observation of conducting filament growth in nanoscale resistive memories." Nature Communications 3, 732 (2012).

24. R. Waser, M. Aono, "Nanoionics-based resistive switching memories." Nature materials 6, 833 (2007).

25. X. Guo, C. Schindler, S. Menzel, R. Waser, "Understanding the switching-off mechanism in $\mathrm{Ag}$ migration based resistively switching model systems." Applied Physics Letters 91, 133513 (2007).

26. H. Xu, Y. Xia, K. Yin, J. Lu, Q. Yin, J. Yin, L. Sun, Z. Liu, "The chemically driven phase transformation in a memristive abacus capable of calculating decimal fractions." Scientific reports 3, (2013).

27. A. H. Zewail, "Four-dimensional electron microscopy." Science 328, 187 (2010).

28. A. Yurtsever, R. M. van der Veen, A. H. Zewail, "Subparticle ultrafast spectrum imaging in 4D electron microscopy." Science 335, 59 (2012). 